\title{
SOLAR AND STELLAR ABUNDANCES OF THE ELEMENTS
}

\author{
By Lawrence H. Aller \\ The University of Michigan Observatory
}

\section{INTRODUCTION}

ASTRONOMERS, nuclear physicists, and cosmologists have long sought to determine the "cosmic" abundances of the elements, i.e. the chemical composition of stars and gaseous nebulae in our part of the galaxy. In this contribution we shall be especially concerned with the composition of the sun which presumably represents the primordial composition of the solar system. The compositions of other stars may differ. A considerable body of astronomical evidence indicates that the stars in the neighborhood of the sun differ in age. Highly luminous stars transmute hydrogen into helium to produce energy at such a great rate that their allotted life span is only a few million years. When we observe a cluster containing brilliant blue stars we can be reasonably sure that we are dealing with a fairly young population. Many such clusters are known, e.g. the Pleiades and the double cluster in Perseus. In a few clusters we appear to be observing the actual formation of stars from the interstellar medium. Other clusters, such as Messier 67, are much older. They contain no luminous stars; in fact, all stars much more massive than the sun have passed on to more advanced stages of their evolution. As a star ages, the hydrogen in its central regions becomes converted into helium until eventually all the energy is generated in a thin shell surrounding an inert contracting core. Then the outer portion of the star becomes distended, the radius of the star increases and its surface temperature falls. Eventually, as the core of the star continues to shrink the helium may become converted into carbon, oxygen, neon and yet heavier elements. The outer portion of the star escapes into space-perhaps in violent ejection as in supernovae, or perhaps smoothly as in such systems as the double star $\alpha$ Herculis. This material may be partly enriched in the heavier elements built up in the stellar interior. As new stars are formed from the interstellar medium they contain a larger portion of heavier elements than did their predecessors. Consequently, the oldest stars should show the greatest ratio of hydrogen to the metals. We expect that the younger the star the greater will be the proportion of metals that it contains. The relationship is not a linear one, however. There is evidence that the young blue stars have very nearly the same composition as the sun insofar as elements such as oxygen, carbon, silicon, magnesium and aluminum are concerned (ALLER, ElSTE and JUGAKU, 1957). We shall not concern ourselves here with these evolutionary problems but shall attempt to see what data can be obtained concerning the probable composition of the solar system at the time the earth was formed. We believe that this composition is presently well illustrated by that of the sun. 
It is just a century since Bunsen and Kirchhoff demonstrated the laws of spectrum analysis that paved the way for the chemical analyses of the sun, the stars, and nebulae. Since then the pioneering qualitative analysis has been replaced by attempts at a quantitative analysis that have proved both stimulating and rewarding. More than thirty years ago Russell (1929) made the first quantitative analysis of the sun. In spite of the poor observational data then available and the rather primitive theory of stellar spectral line formation, his efforts were remarkably successful. For some elements we can do no better than he did, because of our lack of knowledge (even to this day) of the essential physical parameters. Successively more refined methods have been used until at the present time the limiting factor is our knowledge of the transition probabilities of the spectral lines and their sensitivity to density effects (pressure-broadening). The analysis of a star necessarily differs from a conventional spectrochemical analysis. To state the problem in the simplest possible terms: we cannot prepare a sample star in the laboratory and compare it with a real star. We must extract from our observational material both the chemical composition of the star and the physical state of its atmosphere, simultaneously!

\section{THE ATMOSPHERE OF THE SUN}

The sun, which is the only star close enough to appear with an appreciable disk, shows complexities enough to satisfy anyone. The bright white surface or photosphere is mottled with the ephemeral granulation or "rice-grain structure". Above this lies the tenuous chromosphere (best seen as a spiked, fiery envelope at the time of solar eclipses) and the pale white corona which can be observed only with special instruments under conditions of exquisite transparency. These outer solar envelopes, whose time-dependent behavior is of enormous significance to problems of solar-terrestrial relationships and which pose some of the most engaging astrophysical questions, contribute a negligible portion of the total light of the sun.

The radiation from the bright white solar surface provides the familiar dark-line Fraunhofer spectrum of the sun. To a surprisingly high accuracy we can regard this spectrum as produced by an atmosphere consisting of planeparallel stratified layers in which the temperature and pressure increase monotonically with depth. The temperature gradient throughout a large part of the visible atmosphere of the sun can be established from measurements of the decrease in surface brightness from the center to the limb of the sun (the so-called limb darkening). These measurements do not give information on the uppermost strata of the solar photosphere. Here we have to rely on other methods, partly observational and partly theoretical.

In stars other than the sun, the determination of the temperature gradient is more difficult and direct observational methods are usually lacking. In a few eclipsing double star systems information on limb darkening sometimes can be found, and we can get a rough check on our ideas. More often, we have to make some basic postulate, e.g. that the atmosphere is either in radiative equilibrium (i.e. all the energy of the star is transported outward through its atmosphere by radiation) or it is in convective equilibrium, i.e. all the energy is carried upward and outward by convection currents. These two different assumptions lead to somewhat different predictions of the energy distribution 
of the radiation emitted by the star, predictions which are amenable to direct observational checks. As far as we can tell, the energy is transported through the visible layers of stellar atmospheres by radiation, although we must expect that in many stars, zones in convective equilibrium lie directly below the visible strata.

For theoretical studies of stellar spectra the essential quantity is not the geometrical (or linear) depth in the atmosphere, but the optical depth which controls how much of the emission from a given stratum will reach theobserver. Let $\kappa_{\lambda}$ be the absorption coefficient at a given wave length, $\rho$ the density of the material and $\mathrm{d} h$ the element of height. Then the element of optical depth will be

$$
\mathrm{d} \tau_{\lambda}=\kappa_{\lambda} \rho \mathrm{d} h .
$$

Processes involving both the line and continuous spectrum in the sun and stars are usually treated on the assumption that they obey Kirchhoff's law

$$
j_{\nu}=4 \pi B_{v}(T) . \kappa_{v}
$$

i.e. the emission at each frequency is strictly proportional to the absorption coefficient. An exception is the process of electron scattering, which is an important source of opacity in hot super-giant stars. It is independent of wave length and does not depend on the temperature. The principal source of continuous absorption in the visible spectrum of the sun, the photo-dissociation of the negative hydrogen ion, does obey Kirchhoff's law as does the continuous absorption by atomic hydrogen which is the principal contributor to opacity of the atmospheres of stars hotter than the sun. When Kirchhoff's law is obeyed for both the line and continuous spectrum we say that the material is in local thermodynamic equilibrium (LTE).

\section{THE FORMATION OF SPECTRAL LINES}

If a light quantum is scattered by an atom without being changed in frequency, the temperature of the material does not enter into the expression for the rate of energy output for the material. It has been frequently suggested that the strongest lines in the solar spectrum are formed by scattering, and extensive calculations of the shapes and intensities of spectral lines have been carried out on this assumption. Curiously, in spite of extensive, elaborate studies of the profile and total intensity variations of many lines across the disk of the sun, it has not been possible to decide clearly between these two physical mechanisms, scattering or LTE. For the time being we shall assume that the mechanism of LTE prevails. This is not an essential requirement; if instead we prefer to adopt the scattering mechanism we can carry out the appropriate calculations without any essential difficulties.

Let us consider a ray emergent from the disk of the sun making an angle $\theta$ with respect to the outward normal. We define

$$
\mu=\cos \theta \text {. }
$$

We now use $\kappa_{\lambda}$ to denote explicitly the coefficient of continuous absorption defined per gram of stellar material, and $l_{v}$ the coefficient of line absorption. Then the element of optical depth for a point in the line will be

$$
\mathrm{d} \tau_{\nu}=\left(\kappa_{\lambda}+l_{\nu}\right) \rho \mathrm{d} h .
$$


The intensity of an emergent ray will be

$$
I(0, \theta)=\int_{0}^{\infty} B(\xi) \mathrm{e}^{-\xi \sec \theta} \sec \theta \mathrm{d} \xi
$$

where $\xi$ denotes $\tau_{\lambda}$ for the continuum and $\tau_{\nu}$ for a point in the spectrum line. The residual intensity in the line is:

$$
r_{v}=\frac{I_{\nu}(0, \theta)}{I_{\lambda}(0, \theta)}
$$

Across a spectrum line, $l_{v}$ will vary rapidly but $\kappa_{\lambda}$ very slowly. Both will vary with depth in the atmosphere, and we must at the outset know the structure or model of the atmosphere. That is, we are given $T\left(\tau_{0}\right)$, the temperature as a

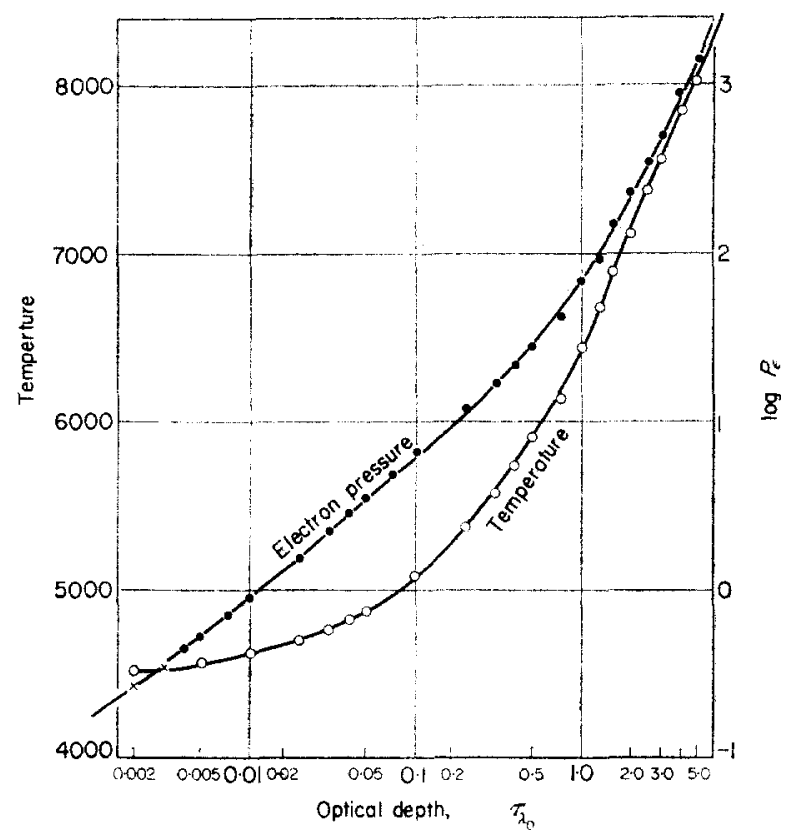

Fig. 1. Model of solar atmosphere.

function of the optical depth at some convenient selected wave length in the continuum. Also, we have the gas pressure as a function of $\tau_{0}, P_{g}\left(\tau_{0}\right)$ and the electron pressure $P_{s}\left(\tau_{0}\right)$. Finally, we must have $\kappa_{\lambda} / \kappa_{\lambda_{0}}$ so we can compute $\tau_{\lambda}\left(\tau_{0}\right)$.

Figure 1 shows the model atmosphere compiled by Elste and used in the quantitative chemical analysis of the sun by GoLDBERG, MülLER and ALLER (1960). As abscissae we employ the optical depth at $\lambda .5000 \AA$. The temperature scale is given at the left, the logarithmic electron pressure scale is at the right. The total gas pressure is not given; it is required only for calculating the absorption coefficients of pressure-broadened lines. Strong spectral lines tend to be formed in the layers with $\tau_{i}<0 \cdot 1$, whereas the weak lines are formed mostly in the 
domain $0 \cdot 1-1 \cdot 0$. With the data included in Fig. 1 and the assumed hydrogen/ metal ratio it is possible to compute $P_{p}\left(\tau_{0}\right), \kappa_{\lambda_{0}}\left(\tau_{0}\right)$ and $\tau_{\lambda}\left(\tau_{0}\right)$.

The line absorption coefficient at any given point on the line profile, $\kappa_{v}$, will depend on a number of factors, only one of which is the abundance of the element in question. These factors are:

(1) The fraction of all the atoms of the element involved that are in a state in which they can absorb the line in question. For a given temperature and electron pressure we can compute this quantity with the aid of the Boltzmann and Saha equations (see e.g. AlLer, 1953, Chapter 4).

(2) The gas kinetic temperature which determines the Doppler width of the absorption coefficient due to the random motion of the atoms. If there is large scale motion of the gas often referred to as turbulence, the absorption coefficient will be further broadened. These effects can be rather complicated and will differ depending on whether the size of the radiating volume element is greater or smaller than the mean free path of a light quantum.

(3) In addition to the Doppler motion as a source of line broadening there is the natural broadening which can become important at large distances from the line centre.

(4) In the atmospheres of dwarf stars like the sun, collisional or pressurebroadening determines the absorption coefficient in the far wings of the line. It is produced by the van der Waals interaction between the radiating atom and the neutral hydrogen and helium atoms.

(5) Interactions between charged particles and radiating atoms produce the interatomic Stark effect. Most atoms are subject to the quadratic Stark effect which results in an absorption coefficient of the same shape as natural broadening. Hence it can be handled by the same formal theory as natural plus Doppler-broadening.

Under the combined influence of Doppler-broadening (including turbulence) and natural plus collisional broadening we may write the expression for the absorption coefficient as

$$
\left.\left.\alpha_{v}=\alpha_{0} H\right) a, u\right)
$$

where $\alpha_{0}$ is essentially the absorption coefficient at the center of the line, which is given by

with

$$
\alpha_{0}=\frac{\pi \varepsilon^{2}}{m c} f \frac{1}{\sqrt{\pi} \cdot \Delta \nu_{0}}
$$

$$
\Delta v_{0}=\frac{\nu_{0}}{c} \sqrt{v_{g}^{2}+v_{\ell}^{2}}, \quad u=\left(\nu-v_{0}\right) / \Delta v_{0}, \quad a=\frac{\Gamma}{4 \pi \Delta \nu_{0}}
$$

where $v_{g}=$ most probable gas kinetic velocity,

$v_{t}=$ turbulent velocity,

$\Gamma=$ damping constant which includes both contributions of radiation damping and collisional damping,

$$
f=\text { Ladenburg } f \text { or "oscillator strength". }
$$

The function $H(a, v)$ is represented by a definite integral which has been evaluated numerically and tabulated as a function of $a$ and $v$. 
Figure 2 shows as an example the absorption coefficient for the $\lambda 36836 p^{3} P_{1-}$ $7 s^{3} P_{0}$ transition in neutral lead. The method of calculation is described on pp. 250-255 of the author's Atmospheres of the Sun and Stars (AlLER, 1953). We take a gas kinetic temperature of $5700^{\circ} \mathrm{K}$. The gas kinetic velocity is $0.67 \mathrm{~km} / \mathrm{sec}$, whereas the turbulent velocity $i$, is taken as $2 \mathrm{~km} / \mathrm{sec}$. We adopt a gas pressure $P_{4}=5.5 \times 10^{4} \mathrm{dyn} / \mathrm{cm}^{2}$ from the model atmosphere at a point $\left(\tau_{0}=0 \cdot 33\right)$ corresponding to $T=5700^{\circ} \mathrm{K}$. Then the collisional damping constant $\Gamma_{\theta}$ is $6.3 \times 10^{8}$, while we adopt a radiative damping constant

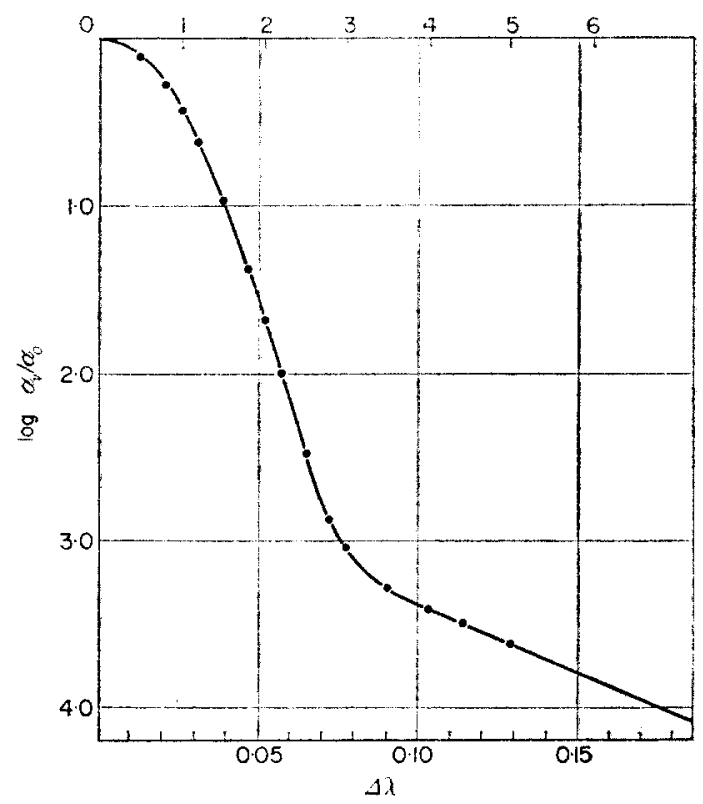

Fig. 2 .

$\Gamma_{\text {rad }}=1 \times 10^{8}$ so that $\Gamma_{\text {total }}=7.3 \times 10^{8}$. Now $\Delta v_{0}=0.572 \times 10^{10}$ with the corresponding $\Delta \lambda_{0}=0.0259 \AA$. Hence $u=38.6 \Delta \lambda(\AA)$. In Fig. 2 we give both $u$ and $\Delta \lambda$ as abscissa with $\log \left(\alpha_{v} / \alpha_{0}\right)$ as ordinate. Notice that for $\Delta \lambda<0.07$ the Doppler motions primarily determine the absorption coefficient. At greater distances from the line center the collisional damping determines the line profile.

It is clear from Eqs. (5) and (6) that the profile and total intensity of the line are going to depend on the relative values of the line absorption coefficient $l_{v}=N_{r, s} \alpha_{v}$ and the coefficient of continuous absorption. In the special case that the ratio

$$
\eta_{\nu}=l_{\nu} / \kappa_{\lambda}
$$

is a constant, independent of optical depth, and the Planckian function can be represented by an approximation of the form

$$
B=B_{0}+B_{1} \tau_{\lambda}
$$


Eqs. (5) and (6) can be solved in closed form and explicit formulae written for the shape of the line profile. This approximation, called the Milne-Eddington approximation, is often used in rough preliminary work.

Precise solutions can likewise be obtained in the Schuster-Schwarzschild approximation wherein it is supposed that the lines are formed in a geometrically thin layer superposed over the photosphere which is presumed to emit a pure continuous spectrum.
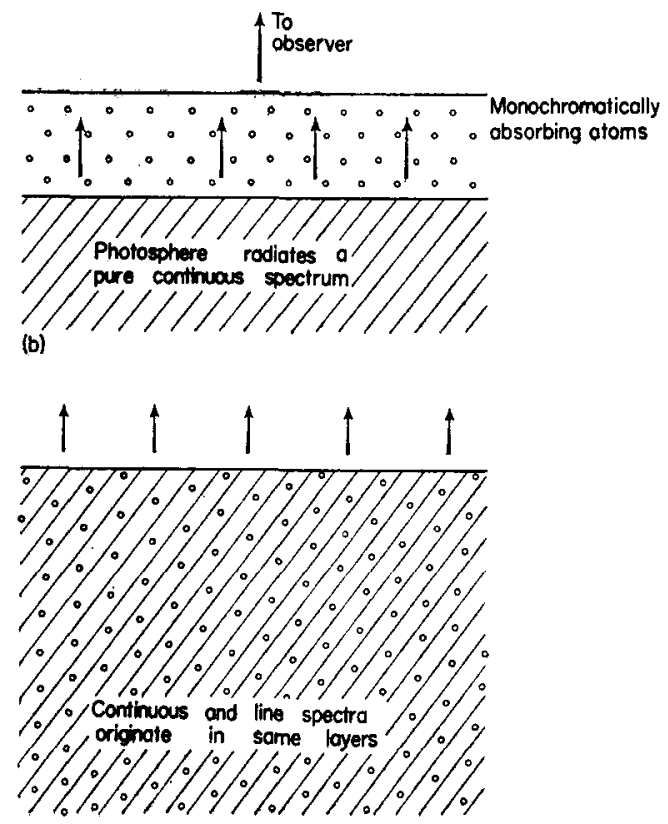

(a)

Fig. 3 (a) Milne-Eddington model. (b) Schuster-Schwarzschild model.

\section{THE CURVE OF GROWTH}

In the first reconnaissance of a stellar spectrum one usually applies what is called the curve of growth procedure. In this technique we adopt the MilneEddington or Schuster-Schwarzschild approximation and calculate the numerical relationship between the total intensity of the line

$$
W_{\lambda}=\int\left(1-r_{\lambda}\right) \mathrm{d} \lambda
$$

i.e. the amount of energy subtracted by the line from the continuum, and the number of atoms acting to produce it.

$$
W_{\lambda} / \lambda=\mathscr{F}\left(N_{r, 8} f \lambda, a\right) .
$$

Here $N_{r, s}$ is the number of atoms capable of absorbing the line in question, $f$ is the oscillator strength for the line, and $a$ expresses the ratio of damping constant to Doppler width (cf. Eq. (8)).

The advantage of the curve of growth is that it permits the observer to handle vast quantities of data quickly. It is invaluable for making comparisons 
between the sun and other stars, for the preliminary analysis of a stellar spectrum, or for comparing stars of normal and abnormal composition. In practical applications one obtains a set of $f$-values, from theory, experiment, or some combination of both, and constructs for each element in each stage of ionization empirical plots of $\log W / \lambda$ against $\log N f \lambda$. Often one makes a separate plot for each multiplet, but sometimes it is possible to construct a curve of growth for a whole group of multiplets, although account must be taken of the appropriate statistical weight and Boltzmann factors if data from several levels of different excitation potentials are combined.

The disadvantages of the curve of growth are that it assumes all lines of all elements in all status of ionization can be treated as though they all originated in a gaseous stratum at a fixed temperature and pressure! This approximation is scarcely valid, for example, if we propose to compare the lines of the carbon monoxide molecule which originate in the uppermost part of the solar atmosphere with the atomic lines of carbon which arise from levels at $7 \cdot 5 \mathrm{eV}$ above the ground state. Also, the curve of growth assumes that all lines of the same total intensity have the same shape. The profile changes with the intensity of the line but there is no provision for the possibility that two lines of the same equivalent width may have different shapes.

\section{THE PRECISE CALCULATION OF LINE INTENSITIES}

The second approximation in the analysis of line intensities is based on the use of model atmospheres. That is, the effects of variation of temperature, pressure and absorption coefficient with depth in the atmosphere are taken into account explicitly. Model atmospheres are constructed on the assumption that the star is in hydrostatic equilibrium and that its outer envelopes are stratified in planeparallel layers. In order to compute a model atmosphere one must select a value of the total energy flux (which defines the effective temperature), the surface gravity, and the chemical composition. One quantity which must be known is the temperature distribution as a function of electron pressure, total pressure or optical depth. The center-to-limb variations of the shapes and intensities of the lines and the darkening toward the limb in the continuous spectrum permit one to determine the temperature distribution in the sun. For other stars we must use some other method, such as the hypothesis of radiative equilibrium, since we observe only the total light of the star.

For most stars the calculation of a model atmosphere is complicated by the extreme non-greyness of the stellar material. That is, the stuff of which a star's atmosphere is composed may be strongly colored in the sense that the coefficient of continuous absorption varies strongly with wave length. In general, we cannot be sure that a particular model atmosphere we calculate applies to any given star. The best procedure is to compute a family or network of model atmospheres. For each one of these we then compute the observable features of the spectrum, i.e. the energy distribution in the continuum, the profiles of certain lines such as the hydrogen lines which are sensitive to surface gravity among the hotter stars, and finally, the intensity ratios of lines of the same element in different stages of ionization. One then compares the theoretical predictions with the observations and interpolates among the models to obtain the best representation of the observations. See e.g. AlLER and JUGaKu (1959).

If one takes into account explicitly the structure of the stellar atmosphere 
and calculates the equivalent widths of the lines making use of the dependence of the line absorption coefficient on the parameter $a$ and the distance from the line center (cf. Eq. (7)), it is possible to obtain an expression of the form

$$
\frac{W_{\lambda}}{\lambda}=\text { const. } N g f \lambda \int_{-\infty}^{+\infty} Z(x) \Psi(Y, a) \Theta(Y, x) G_{\lambda}^{\star}(x) \mathrm{d} x .
$$

In this formulation of the problem, due to PECKER (1951) one computes several functions that depend on the distribution of the relevant atoms in the atmosphere, the total amount of material involved in the production of the line, and the structure of the atmosphere. Specifically, the weighting function $G_{\lambda}^{\star}(x)$ depends only on the wave length and the structure of the atmosphere and not at all on the atom involved. As variable of integration we use the quantity

$$
x=\log \tau_{\lambda}
$$

rather than $\tau_{\lambda}$ itself, as was suggested by ELSTE (1955). Now $Z(x)$ depends on the particular level involved and includes the effects of ionization and excitation throughout the atmosphere, i.e. the variation of the ratio $n_{r, 8} / \Sigma n_{r}$ throughout the atmosphere. The function $Y$ depends on $Z$

$$
Y(x)=c_{1} g_{r, 8} f \lambda N \int_{-\infty}^{+\infty} Z(y)\left(1-\mathrm{e}^{-h \nu / k T}\right) \mathrm{d} x
$$

where $c_{1}$ is a numerical constant, $g_{r, 8}$ is the statistical weight of the level and $N$ is the number of atoms of the given element per gram of stellar material.

The quantity $\Psi(Y, a)$ is essentially Pecker's saturation function which allows for the fact that strong lines are formed predominantly in shallower depths than are weak lines. The function $\Theta(Y, x)$ comes from the fact that the radiation comes from all points on the surface of the star from the center of the disk to the limb. The entire expression under the integral sign in Eq. (13) is called the contribution function. It has to be calculated for the spectral lines of interest in each model atmosphere.

In the sun it is possible to observe the equivalent widths and profiles of lines at preselected points on the disk. Hence, in place of Eq. (13) we have an expression of the form (cf. AlLER, ELSTE and JUGAKU, 1957)

$$
W_{\lambda}^{\mu} / \lambda=\text { const. } N g f \lambda \int_{-\infty}^{+\infty} Z(x) \Psi(a, Y / \mu) g_{\lambda}^{\mu}(x) \mathrm{d} x .
$$

Here $\mu$ is defined by Eq. (3). At the center of the disk $\mu=1$. Here the weighting function $g_{\lambda}^{\mu}(x)$ depends only on the structure of the atmosphere and the point on the disk. Usually, analyses are carried out only for lines at the centre of the disk, although center-to-limb investigations are executed in order to test theories of the structure of the solar atmosphere and mechanisms of line formation.

Quantitative chemical analyses of stellar atmospheres based on model atmospheres have also been carried out by Unsöld's associates at Kiel, namely, Traving (1955), Hunger (1955), and WeIDEMANN (1955). Their analysis postulates that all lines have profiles that can be represented by exactly the same formula, regardless of the point at which they are formed in the atmosphere. 
So far, this type of programme has been carried out for the sun, and for several hotter stars of spectral classes $\mathrm{A}$ and $\mathrm{B}$ with temperatures ranging from 10,000 to $30,000^{\circ} \mathrm{K}$. We confine our attention here to the composition studies that have been carried out by the most refined methods. Although our interest is primarily in the composition of the sun we shall find it necessary to consider also the compositions of other stars as well, notably those whose spectra display the lines of elements not observable in the sun.

Certain light elements such as neon, argon, and fluorine cannot be studied in the sun at all and their abundances with respect to oxygen, nitrogen, etc. can be investigated only in the hotter stars. The reason is that their resonance lines fall in the presently inaccessible far ultra-violet where the intrinsic blending is so severe that even when observations of this spectral region can be obtained with rockets (and eventually we hope with satellites) the spectral lines cannot be disentangled from their neighbors and hence cannot be used for abundance studies. On the other hand, the lines of fluorine, neon, and argon which do fall in the ordinary spectral regions have excitation potentials for their lower levels of $12 \cdot 7,16 \cdot 5$, and $11.5 \mathrm{~V}$, respectively. In the layers in which these lines might be formed in the solar atmosphere, the Boltzmann formula predicts that the population of these levels will be attenuated by factors of the order of $10^{-9}$ to $10^{-13}$. The hypothetical lines arising from these levels would have equivalent widths less than $0.001 \AA$ and would be completely unobservable. Furthermore, it is important to realize that the abundances of elements such as carbon, nitrogen, oxygen, etc. are determined from entirely different lines in the sun and in the hotter stars. In the sun the abundance of oxygen, for example, is found from the infra-red lines of neutral oxygen $O \mathrm{I}$. In the hot stars of spectral class $\mathrm{B}$ (whose temperatures range from $18,000^{\circ} \mathrm{K}$ to $30,000^{\circ} \mathrm{K}$ ) the abundance of oxygen is found from the lines of singly ionized oxygen $\mathrm{O}$ II or even doubly ionized oxygen O III. We compare the relative abundances of the elements that are observed in both the sun and the hot stars and if they are substantially the same we feel entitled to surmise that the solar abundances of the elements not observed in the sun but found in the hot stars could be estimated from the hot star data.

Ten or fifteen years ago such an interpretation of the data was standard operating procedure. At the present time we are more wary. The hot B stars are young objects, only a few million years old, whereas the sun has an age exceeding that of the crust of the earth $\left(4.5 \times 10^{9}\right.$ years $)$. Thus the luminous $B$ stars are presumed to have been formed recently out of the interstellar medium. Since the sun was formed, the composition of this interstellar medium has probably gradually changed. As a star shines, it converts hydrogen into helium and in later stages of its evolution it may subsequently convert some of the helium into heavier elements. Direct observational evidence for this hypothesis of element-building in stars is provided by the presence of the spectral lines of element 43 , technetium, in certain cool, far-evolved stars. In the same stars, abnormally high abundances of elements in the same row of the periodic table, zirconium, molybdenum, etc. are also observed. Presumably, all these elements are formed from metals of the iron group by neutron capture. (See discussions by Burbidge G. and M., F. Hoyle and W. Fowler (1957) and CAMERon $(1959 \mathrm{a}, \mathrm{b})$.) Therefore, we would expect the interstellar medium to be enriched in helium and other "ashes" of hydrogen burning and might anticipate 
the B stars to have a substantially different composition than has the sun. Actually, the amount of this enrichment is unknown, but the evidence we have at present is that the difference in composition between the sun and the younger stars is not very great, i.e. the amount of metals, etc. in the younger stars is probably not more than about twice as much as in the sun. Hence the compositions of these hot stars may still serve as a rough guide for estimating the concentrations of those elements not observed in the sun. A direct determination of the composition of the interstellar medium is difficult, although when the material is caused to shine by fluorescence in the neighborhood of hot stars, estimates of the relative proportions of $\mathrm{H}, \mathrm{He}, \mathrm{O}, \mathrm{N}, \mathrm{Ne}$, etc. can be made. The Orion nebula furnishes an excellent example. From a theoretical analysis of spectrophotometric measurements of line intensities in the Orion nebula, LILLER (1959) and the present writer found substantially the same abundances for the nebula as for the stars. There is some indication that the abundances of certain elements, e.g. chlorine and argon, might be determined more reliably from the nebula than from the stars!

Helium, which is the most abundant element in the universe next to hydrogen, is not observed in the dark-line Fraunhofer spectrum of the sun, although it is found in the chromosphere.* Carbon, nitrogen and oxygen, for which the abundance determinations are based on the greatest number of lines, appear to have closely similar abundances in the B stars and the sun. As for $\mathrm{Mg}, \mathrm{Al}$, $\mathrm{Si}$, and $\mathrm{S}$, one must remark that uncertainties in the $f$-values are such that we cannot regard the differences between the solar and stellar abundances as securely established.

For the elements heavier than argon we must relay on the sun and similar stars. One of the main items of interest in connection with a determination of the quantitative composition of the sun is the possibility of using the solar abundances as the primordial composition of the solar system.

Many years ago it was pointed out by the geochemist GoLDSCHMIDT (1937) that the meteorites provided a much better index of the composition of the solar system (at least insofar as the non-volatile constituents were concerned) than did the crust of the earth. The class of stony meteorites known as chondrites constitutes the vast bulk of these objects and their average composition apparently provides some of the best evidence we have at the moment on the primordial composition of the solar system.

The resemblance between the composition of stony meteorites and that of the sun was noted long ago. More recent refined work has suggested that for certain elements discordances may exist. It is very important to establish whether or not such differences are real or whether they are the effects of errors in the methods of analysis.

Since the manner of formation of meteorites is not known, we might suppose that discrepancies could be explained in terms of different rates of loss of different elements. Abundance deficiencies for volatile elements are easily understood, but differences in abundances of elements with similar chemical properties require careful scrutiny. Urey has pointed out that although the detailed processes involved in the formation of meteorites are not known, elements with closely related chemical and thermodynamical properties should

* An exception is the $\lambda 10830 \mathrm{He} I$ line which is observed sometimes in absorption on the disk, but under conditions that preclude its use for abundance determinations. 
be lost (or retained) in similar proportions. For example, the solar chromium/ iron ratio is given as about ten times the ratio in meteorites. This difference could be explained if the absolute $f$-values were in error by a factor of 3 for each of these metals but in opposite directions! Among other metals for which discordances exist are indium and perhaps lead. If we accept the meteorite abundances with respect to silicon, indium should not be observed in the sun! These questions require further investigation.

Furthermore, the solar data need further analysis. The next step will entail the careful study of particular lines and their analysis by model solar atmospheres on an individual basis. Such a program recently has been initiated by $O$. C. MOHLER and the writer at the McMath Hulbert Observatory of the University of Michigan with the aid of a grant from the National Science Foundation.

A typical situation in the spectrum of the sun is that the line of a relatively rare but strategic element falls on the wing of a strong line of some much more abundant element. For example, a line of lead may fall on the wing of a line of cobalt. Then the wing of the cobalt line forms the "continuum" upon which the lead line is formed.

\section{LEAD IN THE SOLAR SPECTRUM}

The situation may be made clearer by describing a few examples in point. Consider first the four best lines of lead in the solar spectrum. Figs. 4, 5 and

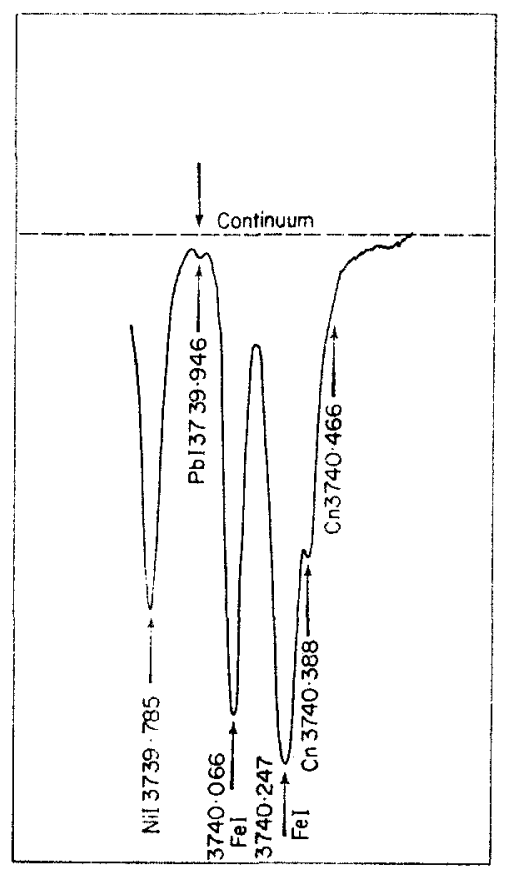

Fig. 4.

6 exhibit tracings made at the center of the solar disk for the new Michigan abundance program. Some tracings were secured with the vacuum spectrograph at Lake Angelus; others were obtained with the Snow telescope at Mt. 


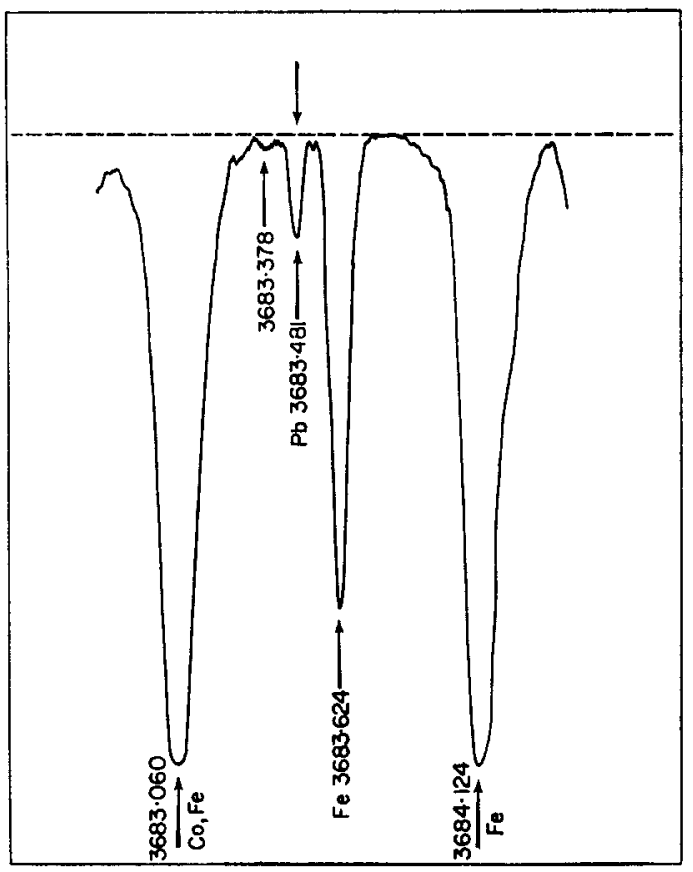

Fig. 5.

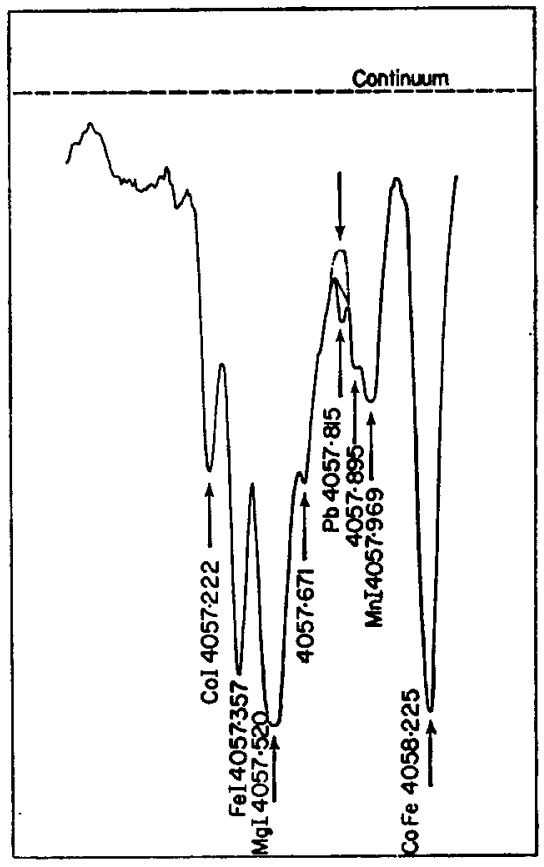

Fig. 6. 
Wilson. The estimated position of the continuum is indicated on each of these tracings. Notice the weak line at 3739.946 between two strong lines of Ni I and Fe I, and the difficulty of estimating the actual position of the background. The relatively strong $\lambda 3683$ line can be measured with apparently considerably greater accuracy than can the other lead lines. The $\lambda .4057 .82$ line is particularly difficult to handle. The dashed curve indicates one possible position of the effective background; the dot-dash curve indicates another possible background.

Figure 7 shows the centre-limb variation of the 3639.526 lead line which is strongly blended with the cobalt 3639.451 line. As we proceed from the center to the limb, i.e. to successively smaller values of $\mu=\cos \theta$, the depths of the lines decrease and the lead line becomes less and less prominent.

\section{PROBLEM OF WEAK LINES AND BLENDS}

In the work of ClaAs in 1951 and the more extensive studies by GoLDBERG, MúlLER and ALLeR (1959) on the abundances of elements in the sun, it is assumed that the weighting function varies slowly with wave length and the influence of blends is taken into account only in an approximate way. It is evident that the next step in the analysis is going to be an extremely laborious one. With abundant elements possessing many lines it is possible to select unblended lines for the construction of curves of growth. Among the rare elements this is no longer possible and one often has to treat the individual lines according to the degree and nature of the blending. In the ultra-violet portion of the spectrum the blending becomes so severe that we no longer see the background continuum, and the local "continuum" is produced largely by the overlap of numerous strong, moderately strong, or even weak lines. This is a problem of actual or intrinsic overlap; no increase of resolution in the spectrograph can eliminate it.

In the first approximation we attempt to allow for the mass effects of these sundry contributors in the following way. Since the source of opacity is presumably the lines of metals and to some extent molecules we examine the distribution of these absorbers with depth in the sun. Let us assume that the empirical absorption coefficient is proportional to the number of absorbers and write

$$
\kappa_{\lambda}(\mathrm{emp})=A_{\lambda} n\left(\tau_{0}\right)
$$

where $n\left(\tau_{0}\right)$ gives the relative number of metallic atoms or molecules as a function of depth. The metal atoms all tend to be concentrated in approximately the same layers whereas the molecules tend to favour the higher levels of the atmosphere.

The intensity of the radiation in the local continuous spectrum at the center of the disk $I_{\lambda}(0,0)$ and the limb darkening $I_{\lambda}(0, \theta) / I_{\lambda}(0,0)$ are also presumed known. We note

$$
I_{\lambda}(0,0)=\int_{0}^{\infty} B\left(\tau_{\lambda}\right) \mathrm{e}^{-\tau \lambda} \mathrm{d} \tau_{\lambda}
$$

where we shall take

$$
\mathrm{d} \tau_{\lambda}=\left[\kappa_{\lambda}\left(\mathrm{H}^{-}\right)+A_{\lambda} n\left(\tau_{0}\right)\right] \rho \mathrm{d} h .
$$

Now $A_{\lambda}$ is found in the following way: we have $T\left(\tau_{0}\right)$ from our model atmosphere 


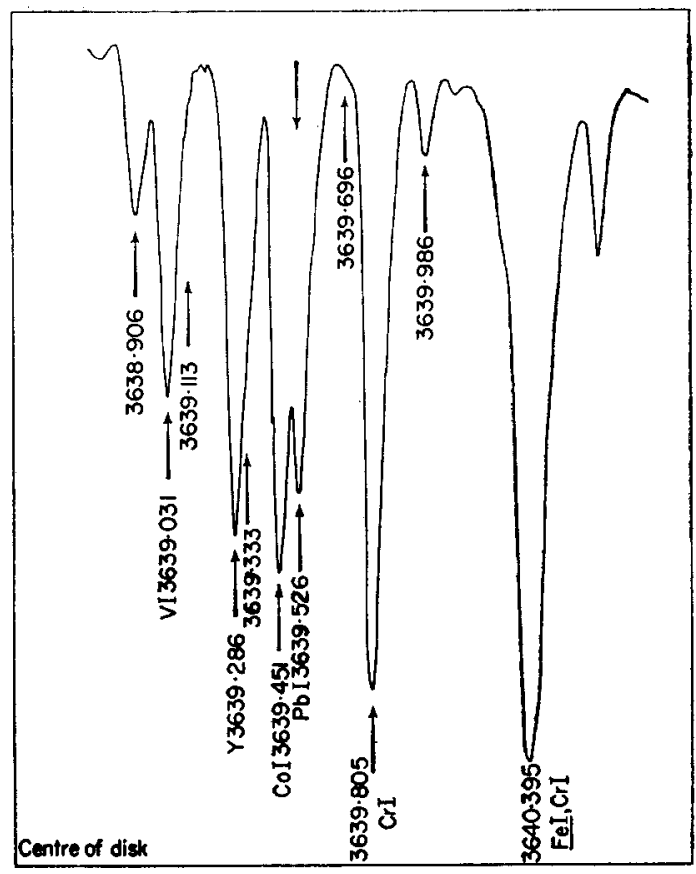

Fig. $7 a$.

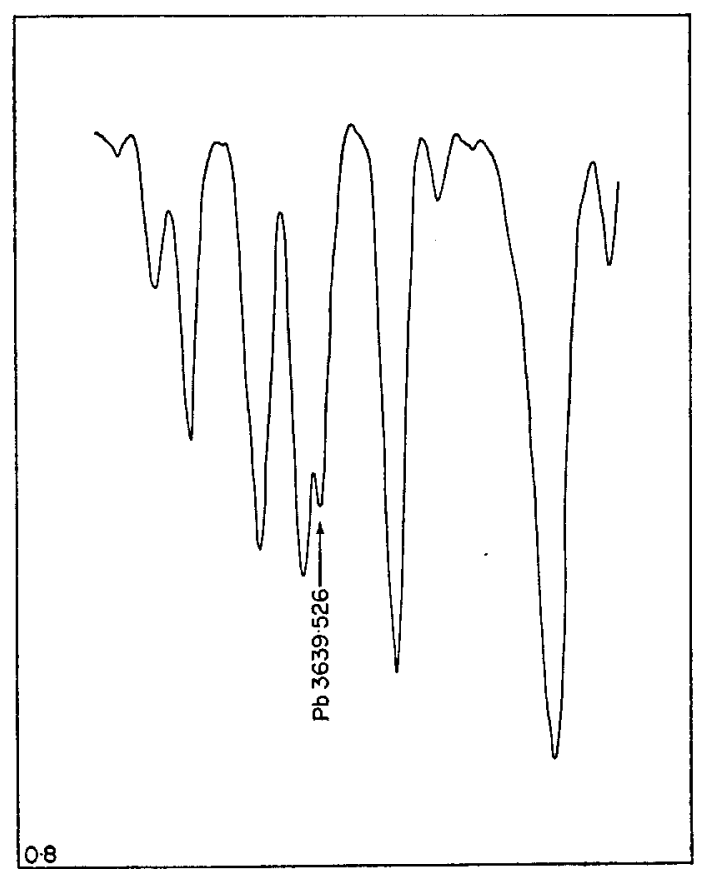

Fig. $7 b$. 


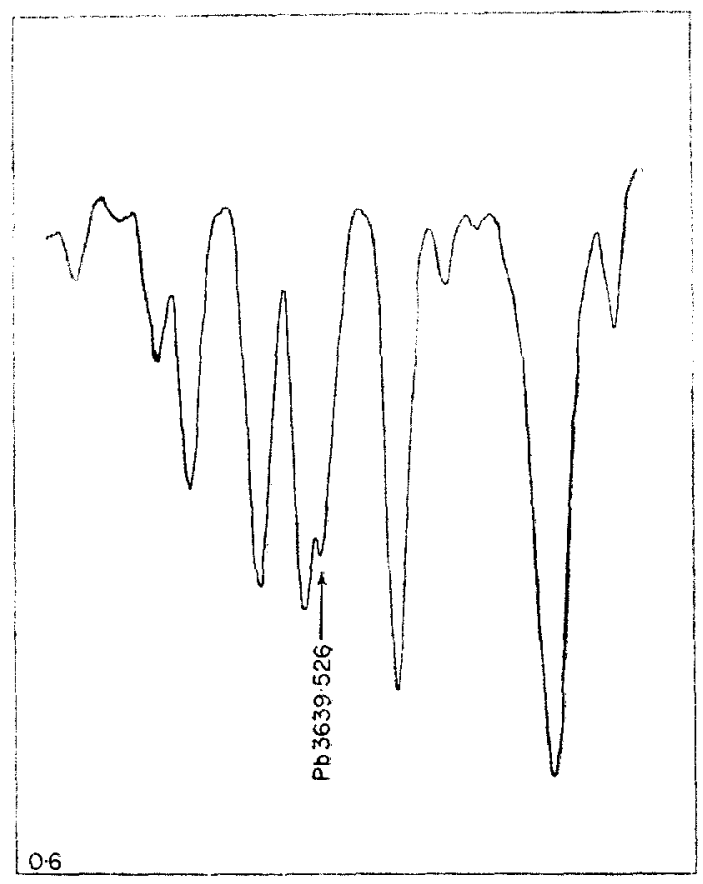

Fig. $7 c$.

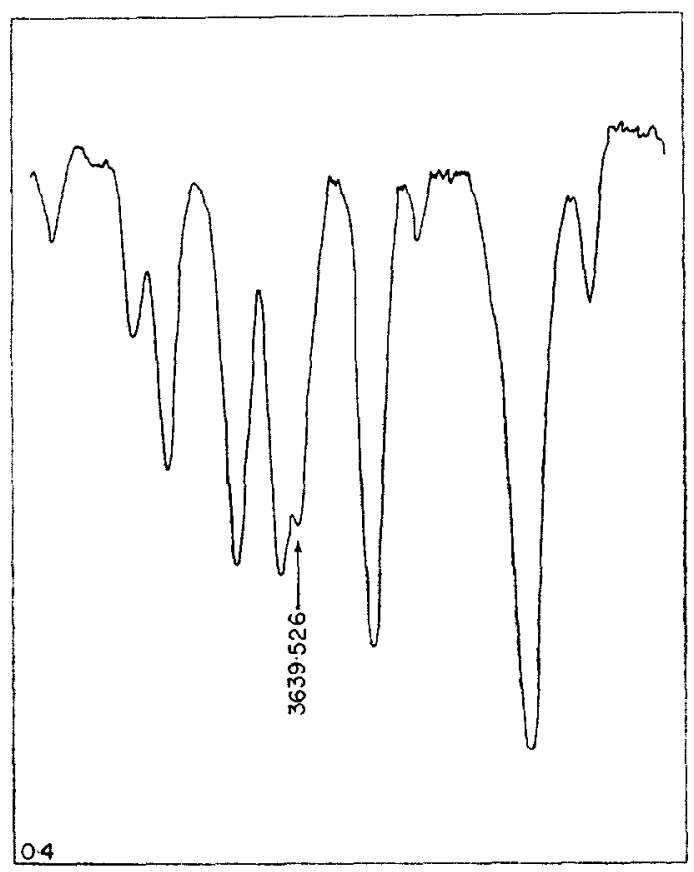

Fig. $7 d$. 
Solar and Stellar Abundances of the Elements

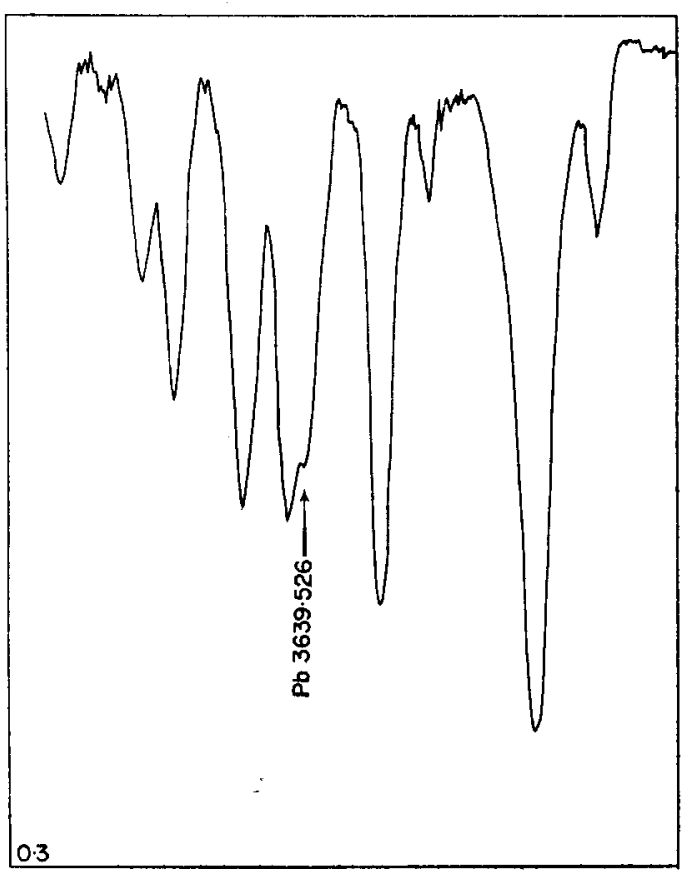

Fig. $7 e$.

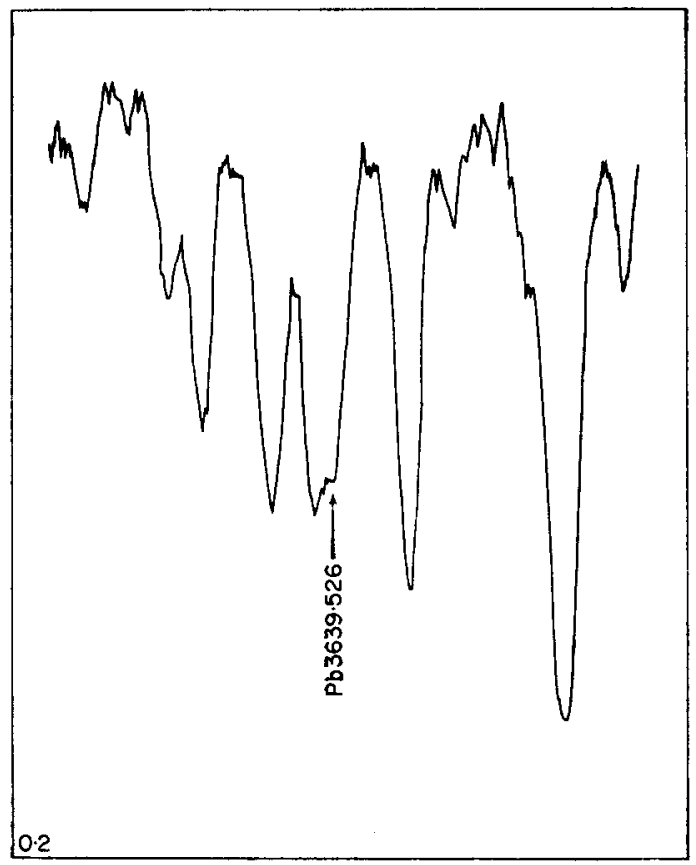

Fig. $7 f$. 
and the Planckian function $B\left(\tau_{\lambda}\right)$ can theretore be given as soon as $\tau_{\lambda}\left(\tau_{0}\right)$ is known. We assume a value of $A_{2}$, compute

$$
\tau_{2}=\int \frac{\kappa_{j}(\mathrm{H})+A, n\left(\tau_{0}\right)}{\kappa_{0}\left(\tau_{0}\right)} \mathrm{d} \tau_{0}
$$

and then evaluate $I_{\lambda}(0,0)$. The process is repeated until the observed $I_{\lambda}(0,0)$ can be reproduced. The calculations can be carried out with $n\left(\tau_{0}\right)$ for the metals or $\eta\left(\tau_{0}\right)$ for molecules and the anticipated limb darkening evaluated. Then from a comparison with the limb darkening measurements $I_{\hat{\lambda}}(0, \theta) / I_{\lambda}(0,0)$ we can see whether the assumed form of the $A_{\ell} n\left(\tau_{0}\right)$, gives the correct depth dependence of the absorption coefficient. When this program is carried out for the $\lambda 3683$ lead line it is found that, although the intensity $I_{\lambda}(0,0)$ can be represented, the limb darkening cannot be reproduced by assuming that the blocking is produced by weak lines. The most important contribution is made by the pressure-broadened wings of moderately strong lines of iron and other metals. Suppose that the blending is caused by such nearby lines of a metal $M$ with a mean lower excitation potential $\bar{\chi}$. Then we must replace $A_{i} n\left(\tau_{0}\right)$ by a term $A_{\lambda} P_{\theta} n\left(M, \bar{\chi}, \tau_{0}\right)$ where $n(M, \bar{\chi})$ is the number of metal atoms at a depth $\tau_{0}$ in the excited level $\bar{\chi}$.

The $\mathrm{Pb} \lambda 3639 \cdot 526$ line which is blended with $\mathrm{Co} \lambda 3639.451$ offers another example. Here the wing of the cobalt line forms the effective "continuum" upon which the lead line is superposed. A rough first approximation could be obtained by assuming a mean line absorption coefficient and adjusting the shape $A_{\lambda}$ to fit the point in the cobalt line profile where the lead line appears. A more rigorous procedure is the following:

The element of optical depth in the true continuum may be written

$$
\mathrm{d} \tau_{\lambda}=\kappa_{\lambda}^{\mathrm{etr}} \rho \mathrm{d} h
$$

where $\kappa_{\lambda}^{\text {eff }}$ includes the mean effect of possible weak unidentified blends and the wings of distant lines. The element of optical depth in the wing of the cobalt line at the point where the lead line falls is

$$
\mathrm{d} \tau_{\lambda}^{P}=\left[\kappa_{\lambda}+l_{P}\left(\Delta \lambda_{P}\right)\right] \rho \mathrm{d} h
$$

while for a point in the lead line itself we have:

$$
\mathrm{d} t_{\lambda}=\left[\kappa_{\lambda}+l_{P}\left(\Delta \lambda_{P}\right)+l_{\nu}\right] \rho \mathrm{d} h
$$

where we neglect the variation of the perturbing cobalt line across the profile of the lcad linc. In a rigorous procedure we would first have to compute the profile of the cobalt line and secure agreement with its observed center-limb variation. Then we would treat this cobalt line as the continuum upon which the lead line is formed and carry out a calculation of its equivalent width. Such a program would require the use of electronic computers and extensive calculations.

Figures 8 and 9 show lines of gallium and of gold with which we have to work. These examples will illustrate the observational difficulties encountered in some of the weaker lines characteristic of the less abundant elements. For 


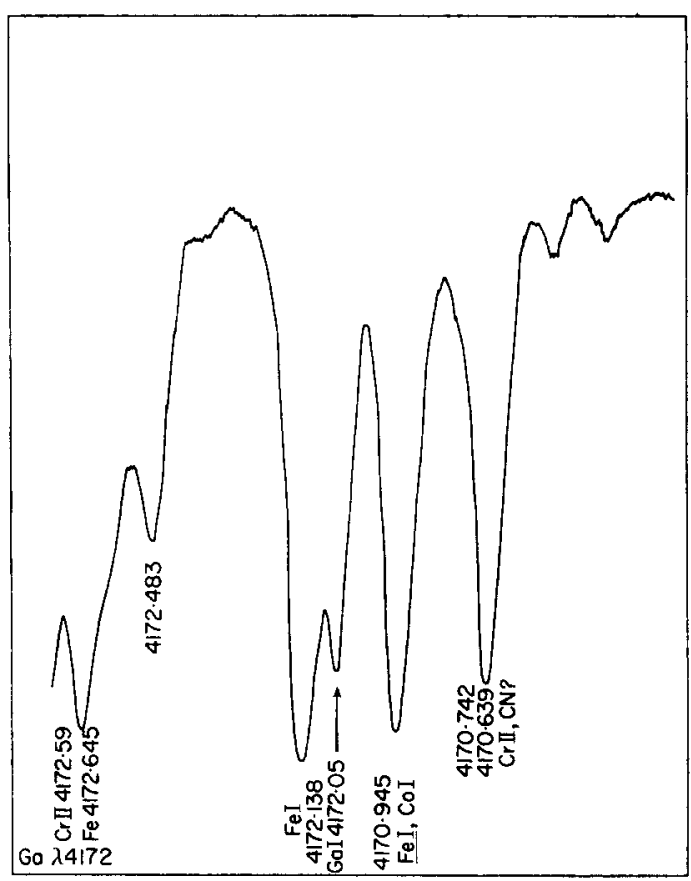

Fig. 8.

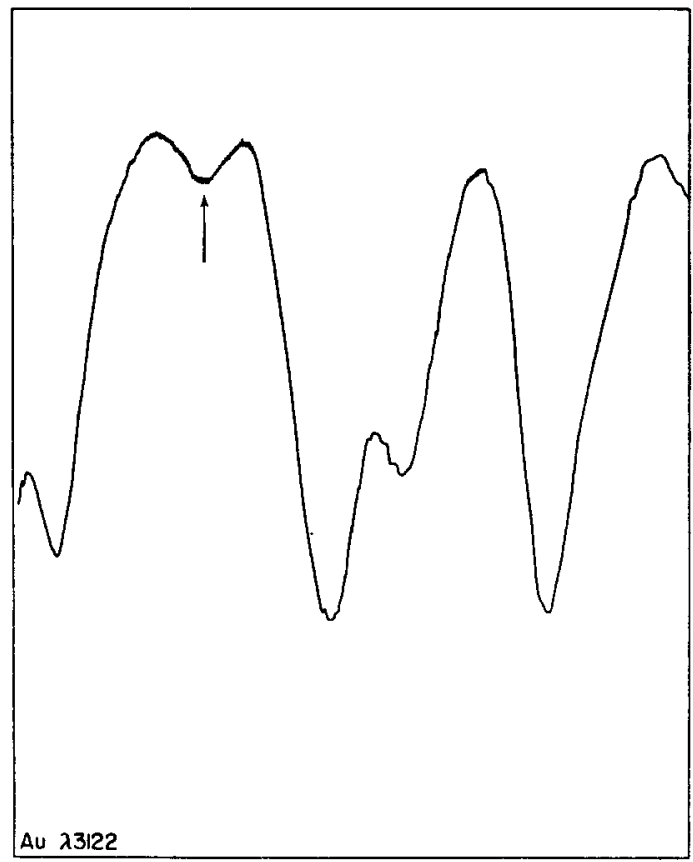

Fig. 9. 
most elements the greatest difficulties are those imposed by our poor information about the $f$-values or transition probabilities.

\section{PROBLEMS OF MORE ABUNDANT ELEMENTS}

For some elements a determination of excellent curves of growth is possible, since the relative transition probabilities are known. Since the absolute transition probabilities are not known or are poorly determined, the actual abundance is correspondingly poorly established. In some instances we attempt to estimate the transition probabilities from theory; for other elements the $f$-values are determined experimentally. A variety of methods are available: the atomic beam method employed by KoPFERMANN and Wessel (1951) and by Bell, Davis, Routly and KING (1958), the whirling fluid arc developed in the laboratory of LOCHTE-HoLTGREven (1952) at Kiel, the luminous shock tube employed, for example, by DOHERTY (1959) in Laporte's laboratory at Michigan. If the vapour pressure of a gas is well enough known as a function of the temperature and if the temperature can be maintained constant in the heated gas, one can use an electric furnace to obtain $f$-values. Usually the experimental difficulties are such that only relative $f$-values can be measured. Sometimes an electric arc method can be used to obtain $f$-values as has been demonstrated by ALLEN (1957). One employs an arc containing metals whose absolute $f$-values are known and also metals whose $f$-values are to be determined. Then, from the relative intensities of the various spectral lines, and an accurate knowledge of the chemical composition of the arc alloy, the temperature of the arc and the $f$-values of the program metals are determined. This is the simplest of the methods but the sharp temperature inhomogeneities in the vapour between the pole pieces imposed a number of difficulties.

For a very few elements moderately good experimental transition probabilities are available and it is possible to obtain reasonably reliable abundances. One of these elements is copper, for which VAN DEN BOLD (1945) measured relative $f$-values. KING and STOCKBARgER (1940) measured absolute $f$-values of the resonance $\lambda 3247$ and $\lambda 3274$ lines with an electric furnace. More recent work by BELL, DAVIS, KING and RouTLY (1958) who used an atomic beam gave results differing from the earlier work by a factor of 1.5 after the latter had been corrected for an error in the vapour pressure measurements. Theoretical calculations by VARSAVSKY (1958), by the BATES-DAMGAARD tables (1949) and by the $f$-sum rule with an $f$-sum of 1.0 give $f$-values that appear to be about twice too large compared with the atomic beam methods.

In the Michigan solar abundance program GoldBERG, MülLeR and ALLER (1960) used eight faint to moderately strong lines in the wave length region $\lambda 4400-\lambda 8100$. The resonance lines $\lambda \lambda$ 3247-3274 not only fall in a part of the solar spectrum where the dependence of the continuous absorption coefficient on the depth is poorly known, but are so strong that a knowledge of the collisional damping constant is necessary in order to make use of them. Such information is not available at present.

The relationship between $\log W / \lambda$ and $\log N g f \lambda$, the curve of growth, where $N$ is the number of copper atoms/gram of solar material, may be calculated from the given structure of the solar atmosphere and the known term structure of copper. The term structure of copper is such that the curve of growth for iron is adequate for copper also. The Michigan workers used the PECKER 
(1951) theory and the iron curve of growth computed by ELSTE (unpublished). As ordinate they employ $\log W / \lambda$ and abscissa the quantity

Here

$$
\log C=\log g f\left[\frac{\lambda(A)}{1000}\right]+\Delta \chi \theta_{0}+\log L_{\lambda}^{\star} .
$$

$$
\Delta \chi=\chi_{\text {ion }}-\chi_{8}
$$

where $\chi_{\text {ion }}$ is the ionization potential and $\chi_{s}$ is the excitation potential of the lower level of the transition involved. Also, $\theta_{0}=5040 / T_{0}$ where $T_{0}$ is some conveniently chosen temperature in the solar atmosphere. Also,

here

$$
L_{\lambda}^{\star}=\int_{-\infty}^{+\infty} Z(x) g_{\lambda}^{0}(x)\left(1-\mathrm{e}^{-h c / \lambda k T}\right) \mathrm{d} x
$$

$$
x=\log _{10} \tau_{\lambda_{0}}
$$

where $\tau_{\lambda_{0}}$ is the optical depth in the continuous spectrum at $\lambda 5000$. The weighting function $g_{\lambda}^{0}(x)$ is computed for the center of the solar disk. Finally, the distribution of metallic atoms capable of absorbing the line in question is given by the function

$$
Z(x)=\frac{1}{\kappa_{\mathrm{eff}}^{\lambda}} \frac{n_{r, 8}}{\Sigma_{n_{r}}} \frac{10^{x-\theta_{0} \Delta x}}{g_{r, 8}} .
$$

Here $g_{r, \theta}$, is the statistical weight of the lower level of the transition involved. The fraction of all atoms capable of absorbing the particular line in question is $n_{r, s} / \Sigma n_{r}$, while $\kappa_{\mathrm{eff}}^{\lambda}$ is the continuous absorption coefficient at the standard wave length.

Table 1. Curve of Growth Data for Copper. (After GoldBERG, MÜLLER and AlLER (1959))

\begin{tabular}{c|c|c|c|c|c|c|c|c|c}
\hline Multiplet & $\Delta J$ & $\lambda(\AA)$ & $\Delta \chi$ & $\log g f$ & $\begin{array}{c}W_{\lambda} \\
(\AA)\end{array}$ & $\log L_{\lambda}^{\star}$ & $\log C$ & $\log W / \lambda$ & \\
\hline $4 s^{2} D-4 p^{2} P^{0}$ & $5 / 2-3 / 2$ & 5105.55 & 6.31 & -2.39 & 82 & -1.864 & $2 \cdot 764$ & -4.794 & $\mathrm{~B}$ \\
& $3 / 2-3 / 2$ & 5700.30 & 6.05 & -2.92 & 14 & -1.904 & 1.982 & -5.607 & $\mathrm{D}$ \\
& $3 / 2-1 / 2$ & 5782.10 & 6.05 & -2.85 & 32 & -1.909 & 2.053 & -5.254 & $\mathrm{D}$ \\
$4 p^{2} P^{0}-5 s^{2} S$ & $1 / 2-1 / 2$ & 7933.16 & 3.92 & -1.41 & 22 & -2.001 & 1.408 & -5.557 & $\mathrm{~B}$ \\
& $3 / 2-1 / 2$ & 8092.64 & 3.89 & -1.09 & 38 & -2.004 & 1.704 & -5.328 & $\mathrm{~B}$ \\
$4 p^{2} P^{0}-4 d^{2} D$ & $3 / 2-5 / 2$ & 5218.21 & 3.89 & -0.63 & 48 & -1.776 & 2.202 & -5.037 & $\mathrm{~B}$ \\
& $3 / 2-3 / 2$ & 5220.09 & 3.89 & -1.58 & 14 & -1.776 & 1.252 & -5.572 & $\mathrm{~B}$ \\
$4 p^{2} P^{0}-6 s^{2} S$ & $1 / 2-1 / 2$ & 4480.39 & 3.92 & -0.94 & 8 & -1.698 & 1.933 & -5.748 & $\mathrm{D}$ \\
\hline
\end{tabular}

Table 1 gives the curve of growth data for copper. The first column gives the multiplet, the second the $J$-values for the lower and upper levels, respectively. The fourth column gives $\Delta \chi$ for the lower level of each transition, while the fifth column tabulates the $\log g f$ values. The $f$-values are taken from the experimental van den Bold measurements combined with the absolute $f$-values obtained from the atomic beam method. The remaining columns give the adopted 
equivalent width, the integral $L_{i}^{\star}$, the abscissa $\log C$, the value of the ordinate $\log W / \lambda$, and information on the blends. Lines of quality $D$ are badly blended. Figure 10 exhibits the final curve of growth. The abundance of copper with respect to hydrogen is found by reading the ordinate of the straight line in Fig. 10 corresponding to $\log C=0$. It is $\log N=-6.96$.

An essentially similar procedure may be followed for iron and a few other elements for which experimental $f$-values are also available. For elements such as chromium, titanium, nickel and cobalt, for which many suitable lines are available, it suffices to give a treatment of the type employed by GoLDBERG, Müller and Aller (1960). The limiting factor lies not in the observations but

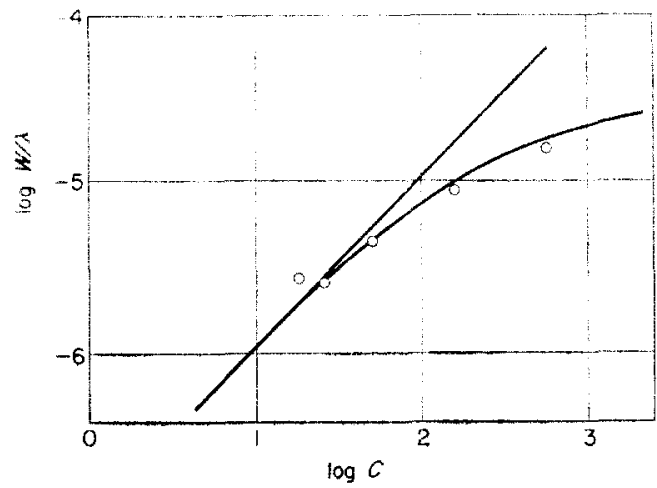

Fig. 10. Curve of growth for copper in the sun. (After Goldburg, MǘlLER and ALLER.)

in our knowledge of the $f$-values. Hence new observations and analyses based on more refined solar models seems scarcely worthwhile, at least until the accuracy of the $f$-values are greatly improved.

Certain other elements, such as lead, gold, ytterbium, are represented by so few lines that detailed observations and analyses directed to the individual circumstances of the blending of each line are in order. In our new program at Michigan we have attempted to carry out detailed studies for a number of elements including $\mathrm{Pb}, \mathrm{Yb}, \mathrm{Au}, \mathrm{In}, \mathrm{Ag}, \mathrm{Ru}, \mathrm{Rh}, \mathrm{Pd}, \mathrm{Zr}, \mathrm{Be}, \mathrm{Li}, \mathrm{Ga}$ and $\mathrm{Ge}$. Lithium and beryllium are important for studies of the structure of the outer layers of the sun. GREENSTEIN (1954) shows that a comparison of the abundances of these elements with respect to, e.g. iron in the sun and on the earth, could give us some clues to the depth at which mixing occurs in the sun since both $\mathrm{Li}$ and $\mathrm{Be}$ are destroyed by nuclear reactions at temperatures well below that of the center of the sun. At the other end of the periodic table, the lead/gold ratio is important to establish the efficacy of certain nuclear processes recently postulated in theories of element building (CAMERoN, 1959b, M. and G. BurBIDGE, Fowler, and HoYle, 1957). Gallium and germanium appear to show opposite abundance ratios in the earth's crust and in meteorites.

Ruthenium, columbium (niobium), rhodium, and palladium are among those elements for which experimental $f$-values are non-existent and whose lines are very faint and often affected by blends. Typically, these lines fall on the wings of strong lines of abundant elements, or at the intensity peaks between strong lines, or are more or less seriously blended with lines of comparable intensity 
due to other elements. All of the lines of Pd fall between $\lambda 3200$ and $\lambda 3600$, the rhodium lines occur between $\lambda 3400$ and $\lambda 3700$, and seven of the ten lines of ruthenium fall between $\lambda 3300$ and 3800 . The ultra-violet spectrum of the sun contains many overlapping lines which makes the analysis difficult (cf. our discussion of $\mathrm{Pb}$ ). New observations from the center to the limb have been obtained but analyses are not yet complete. GoldBerg, Müller and Aller (1959) considered only the lines involving $5 s-5 p$ transitions (i.e. single electron jumps) for which they assumed LS coupling and an $f$-sum of 1.0. They used approximate $L_{\lambda}^{\star}$ functions except for columbium. Most of the lines fall on the straight line portion of the curve of growth. Columbium and ruthenium show a relatively small scatter about a straight line curve of growth, but palladium, whose lines are rather seriously blended, shows a considerable scatter. Hence, in compiling our final list of abundances for the sun we must emphasize again and again the uncertainties imposed by our lack of knowledge of accurate $f$-values. Our solar abundances are no better than the $f$-values on which they are based. With this limitation in mind, let us compare the abundances of the metals in the sun with the corresponding figures for chondritic meteorites.

The meteoritic data are taken from a compilation by MACDONALD (1959) from material by UREY and CRAIG (1953) and by WIIK (1956). Our Table 2 is

Table 2. Comparison of Sun and Chondritic Meteorites

\begin{tabular}{l|c|c|c}
\multicolumn{4}{c}{$\log N$} \\
& $\begin{array}{c}\text { Meteorite } \\
(\mathrm{M})\end{array}$ & $\begin{array}{c}\text { Sun } \\
\text { (S) }\end{array}$ & $\Delta(\mathrm{M}-\mathrm{S})$ \\
& & & \\
\hline & & & \\
$\mathrm{Na}$ & 4.693 & 4.80 & -0.11 \\
$\mathrm{Al}$ & 4.898 & 4.70 & +0.20 \\
$\mathrm{Mg}$ & 5.970 & 5.90 & +0.07 \\
$\mathrm{Si}$ & 6.000 & 6.00 & 0.00 \\
$\mathrm{P}$ & 3.658 & 3.84 & -0.18 \\
$\mathrm{~S}$ & 5.018 & 5.80 & -0.78 \\
$\mathrm{~K}$ & 3.774 & 3.20 & +0.57 \\
$\mathrm{Ca}$ & 4.743 & 4.65 & +0.09 \\
$\mathrm{Ti}$ & 3.356 & 3.18 & +0.18 \\
$\mathrm{Cr}$ & 3.888 & 3.86 & +0.03 \\
$\mathrm{Mn}$ & 3.751 & 3.40 & +0.35 \\
$\mathrm{Fe}$ & 5.853 & 5.07 & +0.78 \\
$\mathrm{Co}$ & 3.344 & 3.14 & +0.20 \\
$\mathrm{Ni}$ & 4.561 & 4.55 & +0.01 \\
& & & \\
\hline
\end{tabular}

the same as MacDonald's Table 7 except that the most recent $f$-value estimates have been incorporated into the solar data. For most elements the agreement is very good. Manganese, cobalt, aluminum, potassium, and particularly iron, are under-abundant in the sun compared with the meteorites. The $f$-values for the first three of these elements are uncertain; the potassium abundance is extremely sensitive to the model of the solar atmosphere. If the uppermost layers are hotter than we have supposed or if the ionization of potassium is greater in these layers, the solar abundance of this element will be increased. The iron abundance is based on $f$-values obtained by the atomic beam method. 
Because of the great astrophysical importance of this metal a considerable effort has been expended to obtain reliable $f$-values and the results by KOPFERMANN and WESSEL (1951) are in good agreement with those by KiNG et al. (1954). We must conclude that the discrepancy between the sun and meteorites is real, although its magnitude may be much smaller than we have supposed if the solar silicon abundance is in error.

MacDonald has remarked that it is possible to construct models of the earth that are in agreement with either the solar or the chondritic meteorite abundances without violating any important data. It is quite clear that if our solar abundances are not grossly in error and if the Earth's interior is constructed from the non-volatile constituents, there is simply not enough iron to provide a pure nickel-iron core of the requisite dimensions and something akin to the nickel-iron-silicate core proposed by MACDONALD and KNOPOFF (1958) is to be expected. On the other hand, if molecular diffusion has altered the composition of the outer layers of the sun in favor of the lighter elements, no agreement is to be expected. The difficulty with appealing to diffusion is that the abundance discrepancies between meteorites and the sun should show a strong dependence on atomic weight.

Sulphur would appear to be more abundant in the sun than in the meteorites. Similar results are found for the young B-type stars, e.g. ALLER and JUGAKU (1959), and the Orion nebula, LILleR and AlLER (1959). The point may be raised that the young stars and the interstellar medium from which they have been formed have an appreciably different composition than has the sun. As previously noted, in the course of $4 \times 10^{9}$ years the interstellar medium has been steadily enriched in heavy elements by material added from the process of element-building in stars. As the stars evolve and manufacture heavy elements out of helium they eventually expand and lose their outer envelopes in space. By this process the interstellar medium is continually being enriched in heavier elements although the rate of addition of material and its exact composition is unknown. The abundances of the other light elements in the early-type stars seems closely comparable with that found in the sun. Recall that the abundances of the noble gases, helium, neon, argon, cannot be found in the sun, and we have to infer them from the data of gaseous nebulae and early-type stars.

Table 3 gives the abundances of the elements in the sun as obtained by GoldBERG, MülLER and ALLER (1960). The starred values are obtained from other stars which show roughly the same abundances as the sun for $\mathrm{C}, \mathrm{N}, \mathrm{O}$, $\mathrm{Mg}, \mathrm{Al}$, and $\mathrm{Si}$, but whose higher temperatures permit them to exhibit also the lines of $\mathrm{F}, \mathrm{Ne}, \mathrm{Cl}$, and $\mathrm{A}$. Sulphur remains an outstanding problem. The B stars and the Orion nebula appear to have a greater concentration of sulphur than has the sun or the chondritic meteorites. Perhaps this increased concentration of sulphur in these objects only reflects the effects of element-building processes in stars.

To summarize, the composition of the sun resembles that of the chondritic meteorites and probably may be taken as the primordial composition of the solar system. Unfortunately, our solar abundance data are incomplete because of the following factors:

(1) Not all elements are represented by lines in the observable solar spectrum. The elements identified in the sun are listed by MOORE (1953). The strongest 


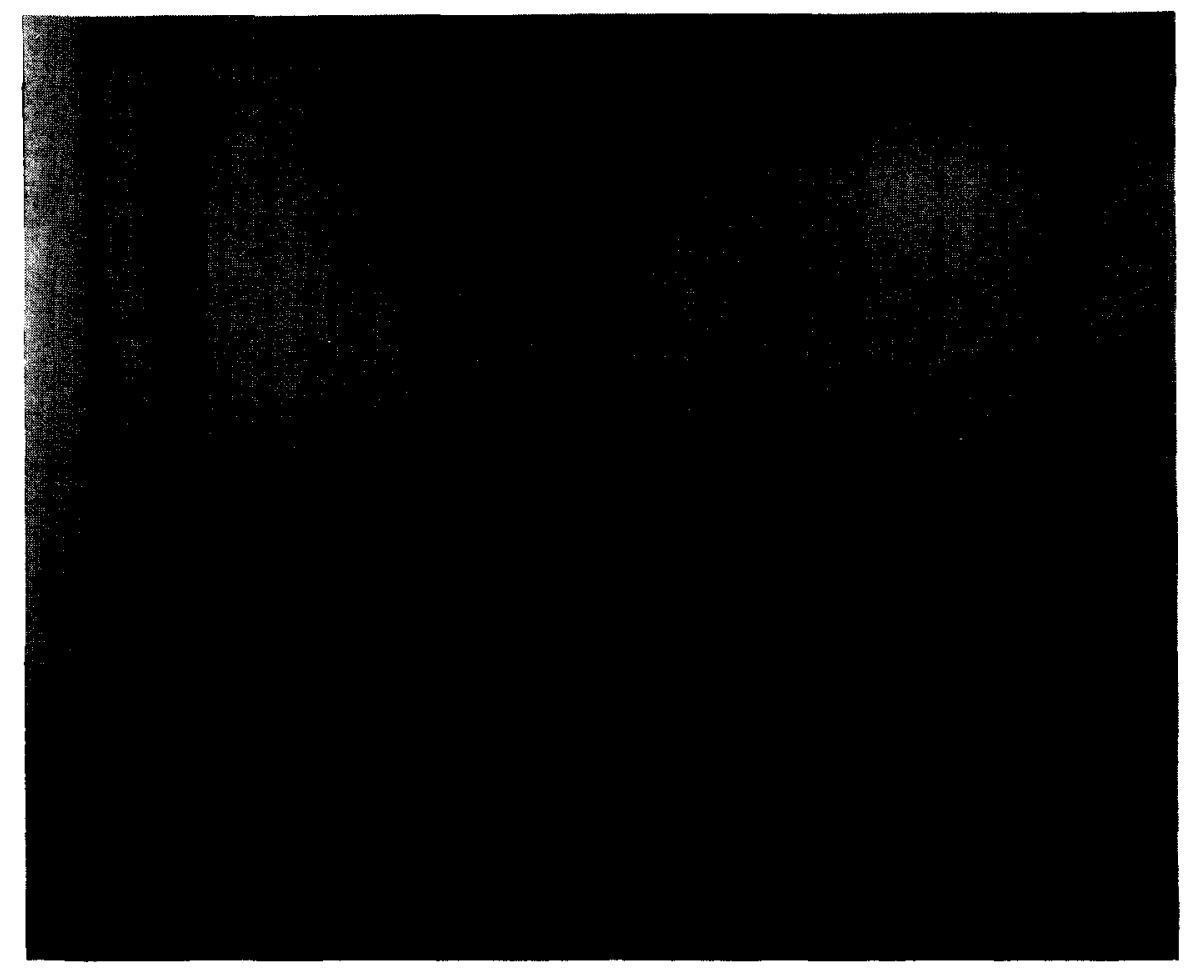

Fig. 11. A portion of the solar spectrum observed with high dispersion. The wiggly character of the lines arise from the Doppler effect of masses of absorbing atoms moving in the line of sight. These moving elementary masses are believed to be associated with the fine structure or "granulation" of the solar photosphere. The fine details are often wiped out by bad seeing, i.e. the effects of unsteadiness in the earth's atmosphere or by the use of a long slit as is necessary in making photoelectric tracings. (McMath-Hulbert Observatory of the University of Michigan.) 
Table 3. Abundances of Elements in the Sun

\begin{tabular}{lc|ll|lr}
\hline \multicolumn{2}{c|}{$\log N$} & \multicolumn{2}{|c}{$\log N$} & \multicolumn{2}{|c}{$\log N$} \\
\hline & & & & \\
$\mathrm{H}$ & 10.50 & $\mathrm{~A}$ & $4 \cdot 1^{*}$ & $\mathrm{Sr}$ & 1.10 \\
$\mathrm{He}$ & $9.65^{*}$ & $\mathrm{~K}$ & 3.20 & $\mathrm{Y}$ & 1.51 \\
$\mathrm{Li}$ & -0.54 & $\mathrm{Ca}$ & 4.65 & $\mathrm{Zr}$ & 0.73 \\
$\mathrm{Be}$ & 0.86 & $\mathrm{Sc}$ & 1.32 & $\mathrm{Cb}(\mathrm{Nb})$ & 0.45 \\
$\mathrm{C}$ & 7.22 & $\mathrm{Ti}$ & 3.18 & $\mathrm{Mo}$ & 0.40 \\
$\mathrm{~N}$ & 6.48 & $\mathrm{~V}$ & 2.20 & $\mathrm{Ru}$ & -0.06 \\
$\mathrm{O}$ & 7.46 & $\mathrm{Cr}$ & 3.86 & $\mathrm{Rh}$ & -0.72 \\
$\mathrm{~F}$ & $5.0^{*}$ & $\mathrm{Mn}$ & 3.40 & $\mathrm{Pd}$ & -0.29 \\
$\mathrm{Ne}$ & $7.5^{*}$ & $\mathrm{Co}$ & 3.14 & $\mathrm{Cd}$ & -0.04 \\
$\mathrm{Na}$ & 4.80 & $\mathrm{Fe}$ & 5.07 & $\mathrm{Ag}$ & -0.86 \\
$\mathrm{Mg}$ & 5.90 & $\mathrm{Ni}$ & 4.55 & $\mathrm{In}$ & -0.34 \\
$\mathrm{Al}$ & 4.70 & $\mathrm{Cu}$ & 3.54 & $\mathrm{Sn}$ & +0.04 \\
$\mathrm{Si}$ & 6.00 & $\mathrm{Zn}$ & 2.90 & $\mathrm{Sb}$ & +0.44 \\
$\mathrm{P}$ & 3.84 & $\mathrm{Ga}$ & 0.86 & $\mathrm{Ba}$ & +0.60 \\
$\mathrm{~S}$ & 5.80 & $\mathrm{Ge}$ & 1.79 & $\mathrm{Yb}$ & +0.03 \\
$\mathrm{Cl}$ & $4.8^{*}$ & $\mathrm{Rb}$ & 0.97 & $\mathrm{~Pb}$ & -0.44 \\
& & & & & \\
\hline
\end{tabular}

* The values represent the "best estimates" taken from the work of GoLDBERG, MüLLER and ALLER (1959), except for the starred values which are adopted from the abundance studies in hotter stars.

lines of $\mathrm{Cs}, \mathrm{Re}, \mathrm{Tl}, \mathrm{Bi}$, and $\mathrm{U}$ fall in the observable part of the solar spectrum, but these elements have not been found. On the other hand, the ultimate lines of the halogens $\mathrm{Cl}, \mathrm{Br}$, and $\mathrm{I}$, the noble gases and $\mathrm{Hg}$, $\mathrm{Te}$, and $\mathrm{Se}$, fall in the inaccessible ultra-violet. Radium and the other radioactive elements are not to be expected. Mercury is not observed in the sun; the lines used by ClaAs (1951) in his abundance determination are probably spurious. Some of these elements ultimately may be observed in the presently inaccessible ultra-violet spectrum of the sun. Helium is in fact observed in the solar chromosphere, and the forbidden lines of [AX] appear in the solar corona. The excitation conditions of the chromosphere and corona deviate so far from thermodynamic equilibrium that these lines cannot be used for abundance determinations.

(2) Some elements such as cadmium, gold, and thorium are represented only by single lines. Elements of low ionization potential, lithium, rubidium and indium, are observed in the sunspot spectrum while boron and fluorine are observed only in the compounds $\mathrm{BH}, \mathrm{MgF}$ and $\mathrm{SrF}$. Ultimately, we will be able to get abundances for boron and fluorine from the lines of their compounds.

(3) For some elements, notably the rare earths, not only are $f$-values lacking but the term analyses are so incomplete that partition functions cannot be computed so the ionization equation cannot be solved. For most elements, as previously noted, the limiting factor is the poor data on the $f$-values. In the future, improved observational data combined with better information on the $f$-values will enable us to obtain more reliable abundances. Observations from satellites of the spectrum of the heretofore inaccessible ultra-violet may add information on those elements whose lines are hidden in this region.

(4) Ultimately, we shall obtain improved models of the solar atmosphere which should take into account the fine structure responsible for the fluctuations exhibited by the granules and the "wiggly" lines (see Fig. 11). 
Finally, we may express the hope that the primordial composition of the solar system may be solved by explorations with space ships. First, we may obtain samples of the Moon, Mercury and of the atmosphere and surfaces of Mars and Venus. The surfaces of Mars and Venus have certainly been affected by their atmospheres and the moon and Mercury may give us no more information than the chondritic meteorites. The asteroids can be sampled; they will have almost certainly the same composition as the meteorites. Probably the true primordial composition of the solar system can be found from analysing samples taken from the satellites of Saturn. These objects are so cold that the substances that are volatile in our part of the solar system-water, ammonia, etc.---are frozen out. An even better sample might be obtained from the satellites of Neptune. It is possible that within the next century significant clues to understanding our own earth will come from space exploration. Just as the astronomer obtains a better idea of the structure of our own galaxy from a study of the Andromeda and other spirals, so may the geophysicists and geochemists obtain a better idea of the structure of our earth from a study of the other planets of our solar system.

\section{REFERENCES}

Aller L. H. (1953) Atmospheres of the Sun and Stars. Ronald Press Company, New York. Aller L. H. (1958) Handbuch der Physik 51, 324.

Aller L. H. and JuGaKU J. (1959) Ap.J. Suppl. 4, 100.

Aller L. H., Elste G. and Jugaku J. (1957) Ap. J. Suppl. 3, 1.

Allen C. W. (1957) Mon. Not. R. Astr. Soc. 117, 622.

Bates D. R. and Damgaard A. (1949) Trans. Roy. Soc. London, A242, 101.

Bell G. D., Davis M. H., King R. B. and Routly P. M. (1958) Ap. J. 127, 775.

Burbidge G. and M., Fowler W. and Hoyle F. (1957) Rev. Mod. Phys. 29, 547.

Cameron A. G. W. (1959a) Ap.J. 130, 429.

Cameron A. G. W. (1959b) Ap.J. 130, 895, 916.

ClaAs W. J. (1951) Utrecht Obs. Researches 12, 1.

DoherTy L. (1959) Thesis, Michigan.

Elste G. (1955) Z. Ap. 37, 201.

Goldberg L., Müller E. and Aller L. H. (1960) Ap. J. Suppl. 45

GoldschmidT V. M. (1937) Geochemische Verteilungsgesetz der Elemente, IX. Die Mengensverhaltnisse der Elemente und der Atom-Arten, Skr. Norske Videnskaps-Akad. I. Mat.Naturv. KI. No. 4.

Greenstein J. L. (1954) Mem. Soc. Roy. Sci. Liege 14, 315.

Hunger K. (1955) Zeit. f. Ap. 36, 42.

KIng R. B. (1954) Proc. Indiana Conf. on Stellar Atmospheres (Ed. Wrubel), p. 41.

King R. B. and Stockbarger D. (1940) Ap. J. 91, 488.

Kopferman H. and Wessel. G. (1951) Z. Phys. 130, 100.

Liller W. and Aller L. H. (1959) Ap. J. 130, 45.

LOCHTE-HOLTGREVEN W. (1952) Observatory 72, 142.

MacDonald G. J. F. (1959) In press.

MacDonald G. J. F. and Knopoff L. (1958) Geophys. J. 1, 284.

Moore C. E. (1953) The Sun (Ed. Kuiper G. P.) p. 186. Univ. of Chicago Press, Chicago.

PeCker J. C. (1951) Ann. Ap. 14, 383.

RusSELl H. N. (1929) Ap.J. 70, 11.

TraVing G. (1955) Z. Ap. 36, 1.

Traving G. (1957) Ibid. 41, 215.

UREY H. C. and Cralg H. (1953) Geochim. Cosmoch. Acta 4, 36.

VAN DEN BOLD H. J. (1945) Thesis, Utrecht.

VARSAVSKY C. (1958) Thesis, Harvard.

Weldemann V. (1955) $Z$. Astrophys. 36, 101.

WIIK H. B. (1956) Geochim. Cosmoch. Acta 9, 279. 


\title{
THE ORIGIN OF THE MAIN GEOMAGNETIC FIELD
}

\author{
By R. Hide and P. H. RoberTs \\ Department of Physics, King's College (University of Durham) \\ Newcastle upon Tyne
}

\section{CONTENTS}

I Introduction . . . . . . . . . . . . $\quad$. 27

I.1 Definition of problem . . . . . . . . 27

I.2 The Earth's interior . . . . . . . $\quad$. 28

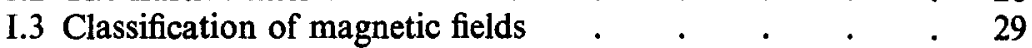

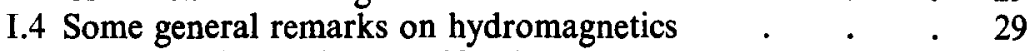

I.5 Theoretical models-classification . . . . . 33

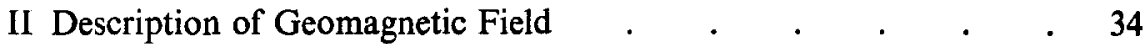

II.1 Magnetic elements $\quad . \quad$. $\quad . \quad$. $\quad . \quad$. 34

II.2 Direction of geomagnetic field $\quad . \quad$. $\quad . \quad$. $\quad . \quad 34$

II.3 Strength of geomagnetic field . . . . . . . 35

II.4 Spherical harmonic analysis . . . . . . . $\quad$. $\quad$. 39

II.5 Magnetic maps; westward drift . . . . . . 40

II.6 The secular variation field.$\quad$. $\quad . \quad$. $\quad . \quad . \quad 41$

II.7 The geomagnetic field in ancient times . $\quad$. $\quad$. $\quad$. 49

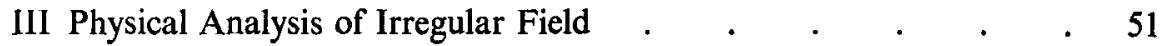

III.1 Limitations of spherical harmonic analyses . . . 51

III.2 Analyses in terms of regional sources in core . . . 52

III.3 Contribution of westward drift of regional sources to secular variation field $. \quad . \quad . \quad . \quad . \quad . \quad . \quad . \quad 58$

III.4 Spectrum of secular variation . $\quad . \quad$. $\quad . \quad . \quad .60$

IV Screening in the Mantle . . . . . . . . 61

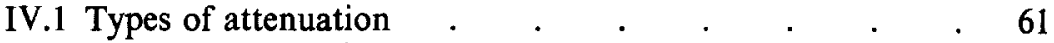

IV.2 Example: Induction in a plane slab . . . . $\quad .62$

IV.3 Screening by spherical shell; time constant of the mantle . 64

V Induction Theories of Main Field . . . . . . 65

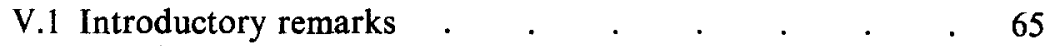

V.2 The dynamo theory of the main field $\quad$. $\quad . \quad$. $\quad . \quad 66$

V.3 The "solid eddy" induction model of the cause of the non-

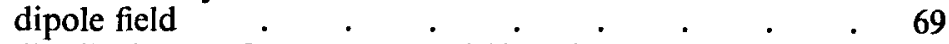

V.4 Bullard's theory of the westward drift and the hydrodynamics of the Earth's core . . . . . . . . 71 
VI Theory of the Secular Variation

VI.I Hydromagnetic models

VI.2 Advection of field

VII Theory of Long Period Changes .

VII.1 Large amplitude oscillations

VII.2 Stability of a simple disk dynamo

VII.3 Behaviour of coupled disk dynamos

Appendices

A. Electromagnetic induction in a plane slab . . . . $\quad .87$

B. Hydromagnetic shear waves . . . . . . 89

Acknowledgments . . . . . . . . . . . 95

References . . . . . . . . . 95 